\title{
Accessing the distance range of interest in biomolecules: Site-directed spin labeling and DEER spectroscopy
}

\author{
Sabine Böhme, Heinz-Jürgen Steinhoff and Johann P. Klare* \\ Department of Physics, University of Osnabrück, Osnabrück, Germany
}

\begin{abstract}
Investigations on the structure and function of biomolecules often depend on the availability of topological information to build up structural models or to characterize conformational changes during function. Electron paramagnetic resonance (EPR) spectroscopy in combination with site - directed spin labeling (SDSL) allow to determine intra- and intermolecular distances in the range from 4-70 $\AA$, covering the range of interest for biomolecules. The approach does not require crystalline samples and is well suited also for molecules exhibiting intrinsic flexibility. This article is intended to give an overview on pulsed EPR in conjunction with SDSL to study protein interactions as well as conformational changes, exemplified on the tRNA modifying enzyme MnmE.
\end{abstract}

Keywords: Site-directed spin labeling, SDSL, double electron-electron resonance, DEER, distance determination, MTSSL, spin label, MnmE

\section{Introduction}

Understanding the function of biomacromolecules requires information about reaction kinetics, structure and conformational dynamics. The structures and therefore also conformational changes associated with biological function are situated in the Ångström to nanometer range, rendering this length scale most important for biomolecular research.

Typically, structures of biomolecules and their complexes are determined by means of X-ray crystallography [10] or high-resolution NMR spectroscopy [8]. Both techniques provide information on the atomistic level, but suffer from serious limitations. Crystallization of the biomolecule implies that it is removed from its native environment and it has always to be questioned if it is in a form that resembles the functional state. Furthermore, crystallization might be difficult or even impossible, especially for membrane proteins, and this method usually fails where the function relies on the flexibility of the structure or weak interactions between two molecules, although recently X-ray techniques became available which allow studying the structural dynamics of proteins in solution [7]. NMR spectroscopy can, besides structural data, also provide information on this flexibility, but is restricted by the size of the biomolecule. Unless sophisticated isotope labeling strategies are used, nowadays this limit is at about $50 \mathrm{kDa}$. Alternatives to NMR and X-ray crystallography are probe-based techniques such as fluorescence resonance energy transfer (FRET) and electron paramagnetic resonance spectroscopy. Both methods are well

\footnotetext{
${ }^{*}$ Corresponding author: Johann P. Klare, Department of Physics, University of Osnabrück, Barbarastrasse 7, D-49069 Osnabrück, Germany. Tel.: +49 541969 2664; Fax: +49 541969 2656; E-mail: jklare@ uos.de.
} 
suited for accessing distances in the nanometer range, dealing with complex structures and high molecular weights as well as providing information on flexible, less ordered structures $[1,11,26]$. Within this class of methods EPR has two major advantages over fluorescence techniques. First, introduction of a spin label into the protein usually causes smaller perturbations of the protein structure and function since the widely used nitroxide side chains are smaller in size than most fluorescence labels. Second, for nitroxides distance distributions rather than mean distances, to which FRET is limited, can be determined. On ground of these benefits, during the past years site-directed spin labeling (SDSL) in combination with EPR spectroscopy has developed as an efficient tool to access the nanometer length scale and to elucidate structure and conformational dynamics of biomolecules. This technique permits the investigation of soluble proteins and nucleic acids as well as membrane proteins, whereby the size and the complexity of the system under investigation are almost arbitrary $[6,14,15,19]$. Furthermore, through the possibility to choose the sample conditions of the studied system as close to the physiological state as necessary, SDSL EPR spectroscopy became a widely applicable tool for investigation of protein structure and dynamics. Continuous wave (cw) EPR spectroscopy yields information about the mobility of the nitroxide side chain, solvent accessibility, the polarity of its immediate environment, and intra- or intermolecular distances up to $20 \AA$ [6]. Using pulse EPR additional information can be obtained by stretching the range of measureable inter-spin distances up to about $70 \AA$.

This article briefly summarizes the basics of distance measurements by EPR on spin-labeled biomacromolecules, with special emphasis on its application to the tRNA modifying enzyme MnmE.

\section{Site-directed spin labeling}

A very well-established method to modify peptides or proteins with paramagnetic spin labels utilizes the reactivity of the sulfhydryl group of the cysteine residues naturally present or introduced via site-directed mutagenesis. This approach requires that the target protein possesses only cysteine residues at the desired sites, and additional perturbing cysteines can be replaced by serines or alanines [6]. Among the various spin labels available the (1-oxyl-2,2,5,5-tetramethylpyrroline-3methyl)methanethiosulfonate spin label (MTSSL) [3] is most commonly used in SDSL studies. It has high sulfhydryl specificity, a small molecular volume, similar to a tryptophan side chain, and a flexible linker between the piperidine-oxyl moiety and the protein backbone usually allows native folding of the proteins. This flexibility results in a large conformational space accessible for the nitroxide ( $4 \AA<d\left(\mathrm{C}_{\beta}-\mathrm{NO}\right)<8 \AA$, depending on the conformation). Therefore, a direct relation of the experimental distances to the properties of the native side chain replaced by the spin label is not possible, but simulation techniques like molecular dynamics simulation or rotamer library approaches can be used to account for this issue. Besides MTSSL, a variety of other different nitroxide radical compounds are commercially available, comprising longer or sterically more demanding linkers [18].

\section{Distance determination with EPR}

Several techniques have been developed to cover different distance ranges. Interspin distances in the range from $6-20 / 25 \AA$ [25] can be achieved by a detailed line shape analysis of cw EPR spectra [28]. Long-range distances $(\sim 20-70 \AA)$ are obtained my means of pulse EPR techniques, namely double electron-electron resonance (DEER) spectroscopy (also named pulse electron double resonance - 
PELDOR) $[2,24,26]$ or double quantum coherence (DQC) [4,5]. These techniques are complemented by exchange EPR for the short distance range (4-10 ̊) [28].

DEER/PELDOR spectroscopy was originally introduced in the 1980s [22,23]. During the past few years this technique has been further developed $[17,20,24]$ and applied to elucidate the structure and function of RNA, soluble proteins and membrane proteins [26]. The technique is based on the detection of the modulation of the echo amplitude of a spin population caused by the dipolar interaction with another spin population, which has been excited by a microwave pulse of different microwave frequency. Herein, the frequency $\nu$ of this modulation is inversely proportional to the cube of the distance $r$ between the two spin labels:

$$
\nu(r)=\frac{52.04 \mathrm{MHz} \mathrm{nm}^{-3}}{r^{3}} .
$$

In the case of a distance distribution rather than a single distance consequently the overall signal is the superposition of modulations of different frequencies. Extraction of distances and distributions from the DEER time domain data is then usually carried out by regularization methods, in particular Tikhonov regularization [9], but can also be achieved by model-based approaches, for example, assuming the overall distance distribution being composed of a single or multiple Gaussian distributions [12]. Both approaches have been implemented in the widely used Matlab software package DeerAnalysis developed by Gunnar Jeschke [16].

A very recent example for the application of DEER to yield insight into the structural and functional properties of a biomolecule is given in the following chapter.

\subsection{The tRNA modifying enzyme MnmE}

The GTP hydrolyzing protein MnmE, which is involved in the modification of the wobble position of certain tRNAs [27], belongs to the expanding class of $\mathrm{G}$ proteins activated by nucleotide-dependent dimerization (GADs) [13]. The crystal structure of MnmE suggested the protein to be a multidomain protein with a central helical part in which a canonical Ras-like domain is inserted, and an N-terminal tetrahydrofolate-binding unit. MnmE was predicted to form a dimer in solution, in which the two G domains are separated with a distance of about 50 A between the two P-loops (see Fig. 1A) [27]. Furthermore, a $\mathrm{G}$ domain dimerization had been proposed based on biochemical data and on the crystal structure of the isolated $\mathrm{G}$ domains in complex with the GTP hydrolysis transition state mimic GDP $\cdot \mathrm{AlF}_{x}$. So far, neither the structural model of the full-length MnmE dimer nor association of the $\mathrm{G}$ domains in the context of the full-length dimer had been proven directly.

In a study employing DEER distance measurements between spin labels positioned in the MnmE $\mathrm{G}$ domain and in the $\alpha / \beta$ dimerization domain both issues were addressed [21]. The data for positions S278 and E287 in the G domains (see Fig. 1A) are shown in Fig. 1B. In the apo state without any nucleotide bound, the two spin labels in the dimer exhibit a distance of $55 \AA$ for E287R1 (R1 denotes the spin label side chain) whereas the analysis of position S278 reveals a large distribution from $25 \AA$ to $50 \AA$ A. Upon binding of GTP (here GppNHp, a non-hydrolysable GTP analogue was used) an additional distance centered at $27 \AA$ (S278R1)/37 $\AA$ (E287R1) appears, contributing about $30 \%$ to the distance distribution. This is evidence for an equilibrium between the open conformation with distant $\mathrm{G}$ domains and a closed conformation, where the G domains are in close proximity. Upon GTP hydrolysis, mimicked with the transition state analogue GDP $\cdot \mathrm{AlF}_{x}$, the distance distribution shows a single population maximum at $28 \AA$ (S278R1)/36 $\AA$ (E287R1), indicating a shift of the equilibrium fully towards the 


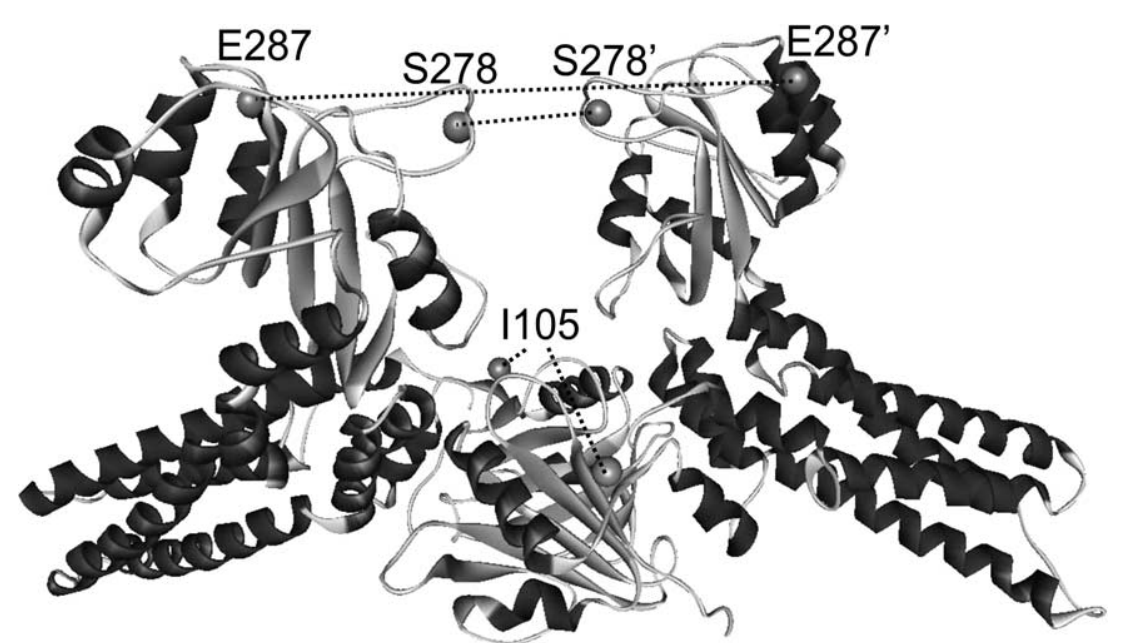

(A)

\section{E287R1}
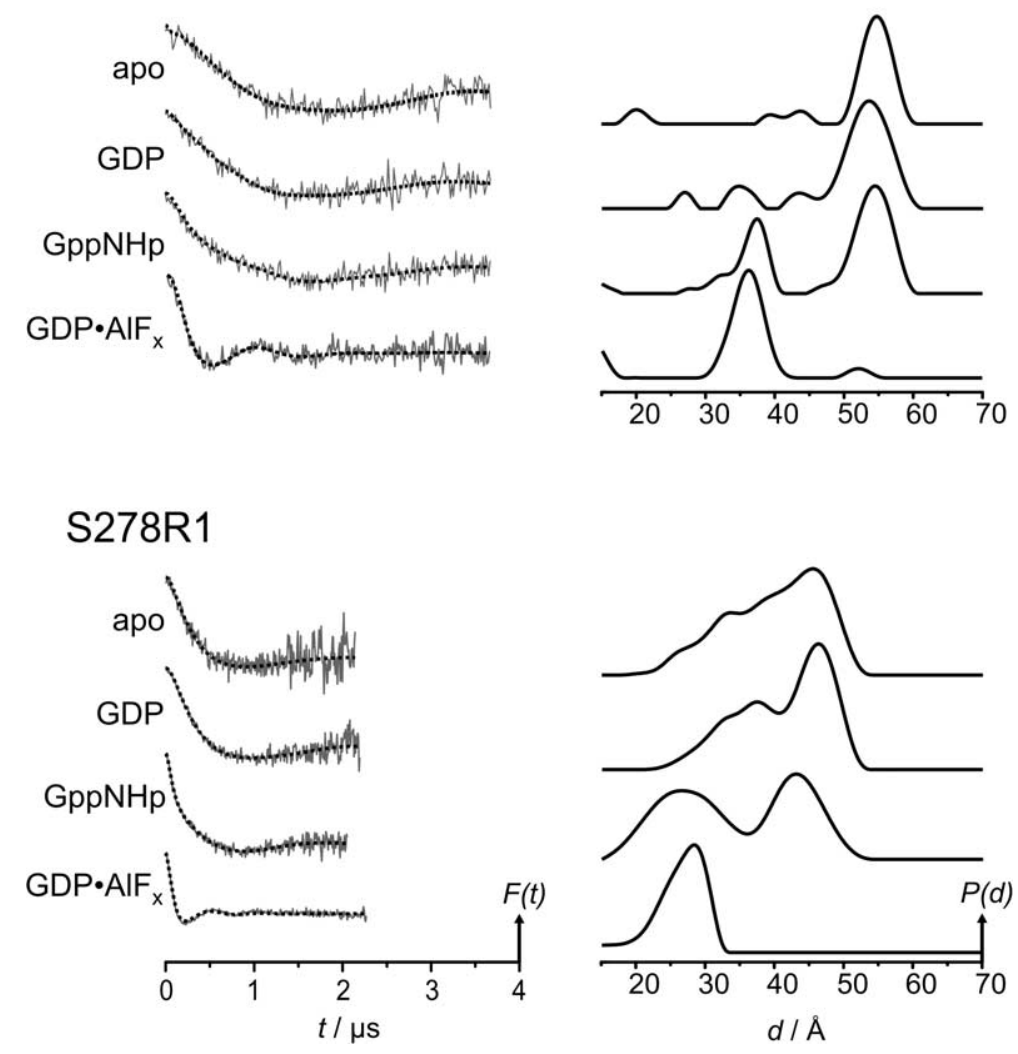

(B)

Fig. 1. (A) Structural model of the MnmE dimer. Positions of residues which were mutated to Cys and spin labeled are indicated as gray spheres, with dashed lines showing distances between residues in the open conformation of the protein. (B) DEER characterization of nucleotide-dependent domain movements for MnmE mutant E287R1and S278R1. Left panel: background

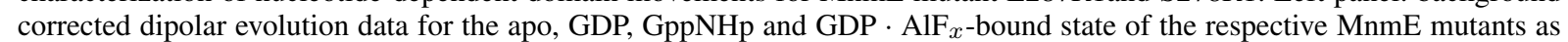
indicated. Right panel: Distance distributions obtained by Tikhonov regularization. 
closed state. After release of the $\gamma$-phosphate group, i.e. with solely GDP present in the nucleotide binding pocket, again a long distance centered at $53 \AA$ for position E287 and a broad distance range from 25-50 A for position S278 are found, almost identical to the observation for the apo state. In addition, spin labels attached at position I105 in the $\alpha / \beta$ dimerization domain showed no significant distance changes during the GTPase cycle, indicating that the initial dimerization interface is largely preserved despite the large $\mathrm{G}$ domain movements (data not shown). Furthermore, a dependency of the GTPase activity and consequently of the $\mathrm{G}$ domain motion on the presence of specific cations could be fully corroborated by the DEER analysis performed in this study.

\section{References}

[1] R.V. Agafonov, I.V. Negrashov, Y.V. Tkachev, S.E. Blakely, M.A. Titus, D.D. Thomas and Y.E. Nesmelov, Structural dynamics of the myosin relay helix by time-resolved EPR and FRET, Proceedings of the National Academy of Sciences of the USA 106 (2009), 21625-21630.

[2] J.E. Banham, C.M. Baker, S. Ceola, I.K. Day, E.J.J. Groenen, C.T. Rodgers, G. Jeschke and C.R. Timmel, Distance measurements in the borderline region of applicability of CW EPR and DEER: a model study on a homologous series of spin-labelled peptides, Journal of Magnetic Resonance 191 (2008), 202-218.

[3] L.J. Berliner and J. Grunwald, A novel reversible thiol-specific spin label: papain active site labeling and inhibition, Analytical Biochemistry 119 (1982), 450-455.

[4] P.P. Borbat and J.H. Freed, Multiple-quantum ESR and distance measurements, Chemical Physics Letters 313 (1999), $145-154$.

[5] P.P. Borbat, H.S. Mchaourab and J.H. Freed, Protein structure determination using long-distance constraints from doublequantum coherence ESR: study of T4 lysozyme, Journal of the American Chemical Society 124 (2002), 5304-5314.

[6] E. Bordignon and H.-J. Steinhoff, Membrane protein structure and dynamics studied by site-directed spin labeling ESR, in: ESR Spectroscopy in Membrane Biophysics, M.A. Hemminga and L.J. Berliner, eds, Springer-Verlag, New York, 2007, pp. 129-164.

[7] M. Cammarata, M. Levantino, F. Schotte, P.A. Anfinrud, F. Ewald, J. Choi, A. Cupane, M. Wulff and H. Ihee, Tracking the structural dynamics of proteins in solution using time-resolved wide-angle X-ray scattering, Nature Methods 5 (2008), 881-886.

[8] J. Cavanagh, W.J. Fairbrother, A.G. Palmer, M. Rance and N.J. Skelton, Protein NMR Spectroscopy, Academic Press, New York, 2007.

[9] Y.W. Chiang, P.P. Borbat and J.H. Freed, The determination of pair distance distributions by pulsed ESR using Tikhonov regularization, Journal of Magnetic Resonance 172 (2005), 279-295.

[10] J. Drenth, Principles of Protein X-Ray Crystallography, 3rd edn, Springer-Verlag, New York, 2007.

[11] M. Edidin, Fluorescence resonance energy transfer: techniques for measuring molecular conformation and molecular proximity, in: Current Protocols in Immunology, Unit 18.10, Wiley, Hoboken, 2003, Chapter 18.

[12] P.J. Fajer, L. Brown and L. Song, Practical pulsed dipolar ESR (DEER), in: ESR Spectroscopy in Membrane Biophysics, M.A. Hemminga and L.J. Berliner, eds, Springer-Verlag, New York, 2007, pp. 95-128.

[13] R. Gasper, S. Meyer, K. Gotthardt, M. Sirajuddin and A. Wittinghofer, It takes two to tango: regulation of G proteins by dimerization, Nature Reviews Molecular Cell Biology 10 (2009), 423-429.

[14] W.L. Hubbell, A. Gross, R. Langen and M.A. Lietzow, Recent advances in site-directed spin labeling of proteins, Current Opinion in Structural Biology 8 (1998), 649-656.

[15] W.L. Hubbell, H.S. Mchaourab, C. Altenbach and M.A. Lietzow, Watching proteins move using site-directed spin labeling, Structure 4 (1996), 779-783.

[16] G. Jeschke, V. Chechik, P. Ionita, A. Godt, H. Zimmermann, J.E. Banham, C.R. Timmel, D. Hilger and H. Jung, Deer analysis 2006 - a comprehensive software package for analyzing pulsed ELDOR data, Applied Magnetic Resonance 30 (2006), 473-498.

[17] G. Jeschke and Y. Polyhach, Distance measurements on spin-labeled biomacromolecules by pulsed electron paramagnetic resonance, Physical Chemistry Chemical Physics 9 (2007), 1895-1910.

[18] J.P. Klare and H.-J. Steinhoff, Spin labling EPR, Photosynthesis Research 102 (2009), 377-390.

[19] C.S. Klug and J.B. Feix, Methods and applications of site-directed spin labeling EPR spectroscopy, in: Methods in Cell Biology. Biophysical Tools for Biologists, Vol. 1, J.J. Correia and H.W. Detrich, eds, Academic Press, New York, 2008, pp. 617-658. 
[20] R.E. Martin, M. Pannier, F. Diederich, V. Gramlich, M. Hubrich and H.W. Spiess, Determination of end-to-end distances in a series of TEMPO diradicals of up to $2.8 \mathrm{Nm}$ length with a new four-pulse double electron-electron resonance experiment, Angewandte Chemie - International Edition 37 (1998), 2833-2837.

[21] S. Meyer, S. Böhme, A. Krüger, H.-J. Steinhoff, J.P. Klare and A. Wittinghofer, Kissing G domains of MnmE monitored by X-ray crystallography and pulse EPR spectroscopy, PLoS Biology 7 (2009), e1000212.

[22] A.D. Milov, A.G. Maryasov and Y.D. Tsvetkov, Pulsed electron double resonance (PELDOR) and its applications in free-radicals research, Applied Magnetic Resonance 15 (1998), 107-143.

[23] A.D. Milov, K.M. Salikohov and M.D. Shirov, Application of ELDOR in electron spin echo for paramagnetic center space distribution in solids, Fizika Tverdogo Tela 23 (1981), 975-982.

[24] M. Pannier, S. Veit, A. Godt, G. Jeschke and H.W. Spiess, Dead-time free measurement of dipole-dipole interactions between electron spins, Journal of Magnetic Resonance 142 (2000), 331-340.

[25] M.D. Rabenstein and Y.-K. Shin, Determination of the distance between two spin labels attached to a macromolecule, Proceedings of the National Academy of Sciences of the USA 92 (1995), 8239-8243.

[26] O. Schiemann and T.F. Prisner, Long-range distance determinations in biomacromolecules by EPR spectroscopy, Quarterly Reviews of Biophysics 40 (2007), 1-53.

[27] A. Scrima, I.R. Vetter, M.E. Armengod and A. Wittinghofer, The structure of the TrmE GTP-binding protein and its implications for TRNA modification, EMBO Journal 24 (2005), 23-33.

[28] H.-J. Steinhoff, N. Radzwill, W. Thevis, V. Lenz, D. Brandenburg, A. Antson, G.G. Dodson and A. Wollmer, Determination of interspin distances between spin labels attached to insulin: comparison of electron paramagnetic resonance data with the X-ray structure, Biophysical Journal 73 (1997), 3287-3298. 


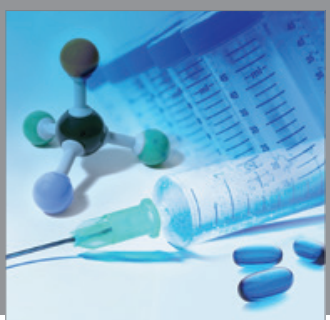

International Journal of

Medicinal Chemistry

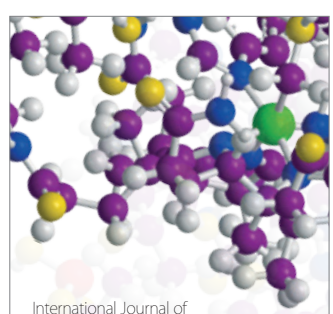

Carbohydrate Chemistry

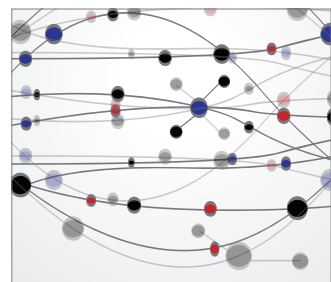

The Scientific World Journal
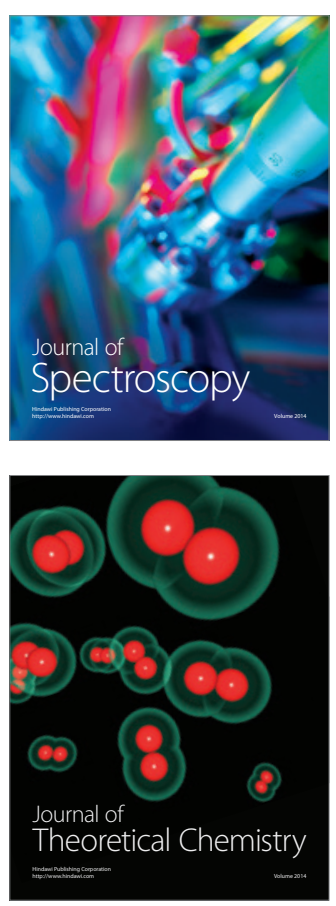
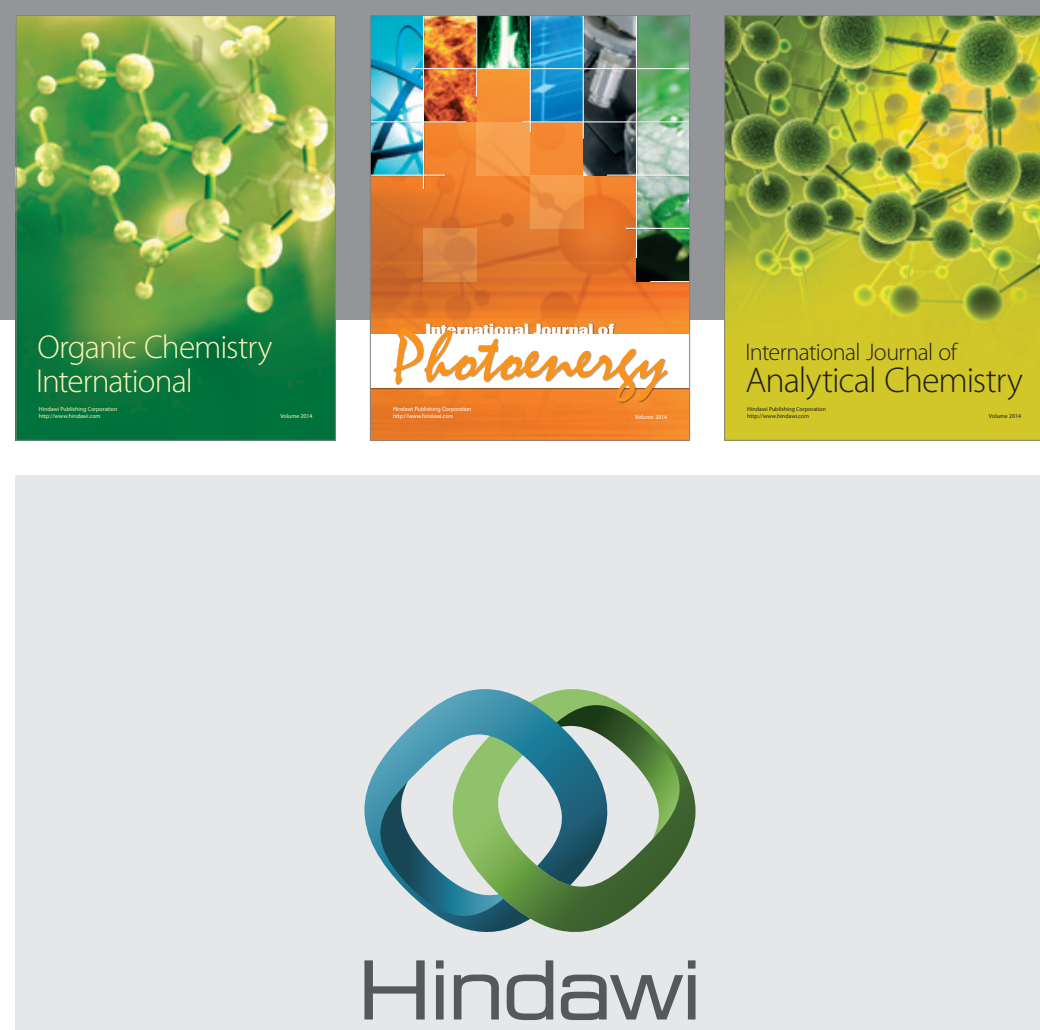

Submit your manuscripts at

http://www.hindawi.com
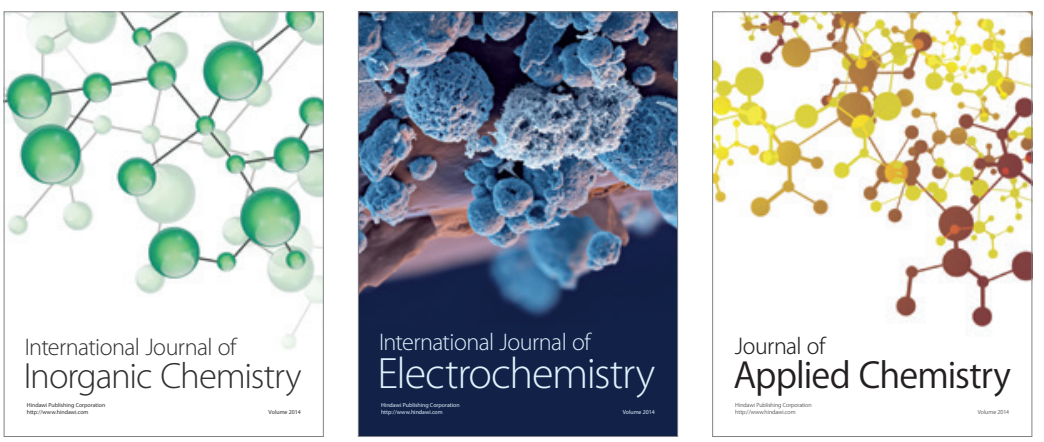

Journal of

Applied Chemistry
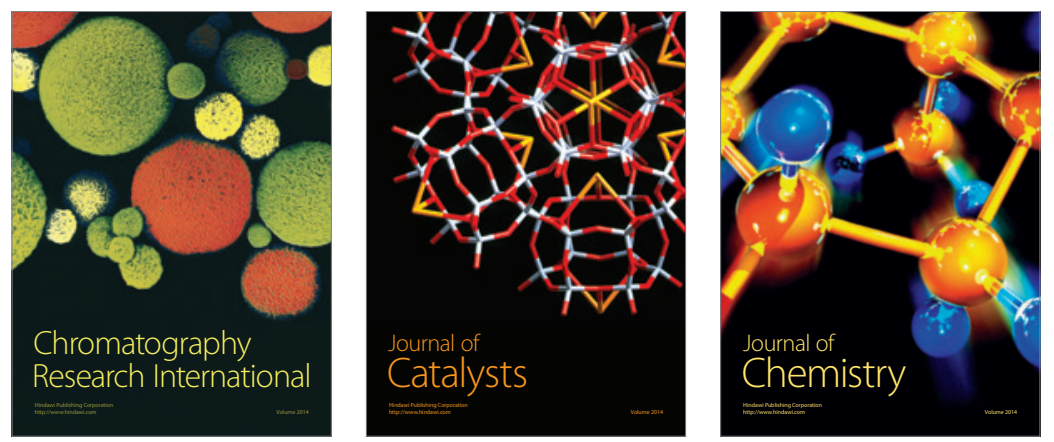
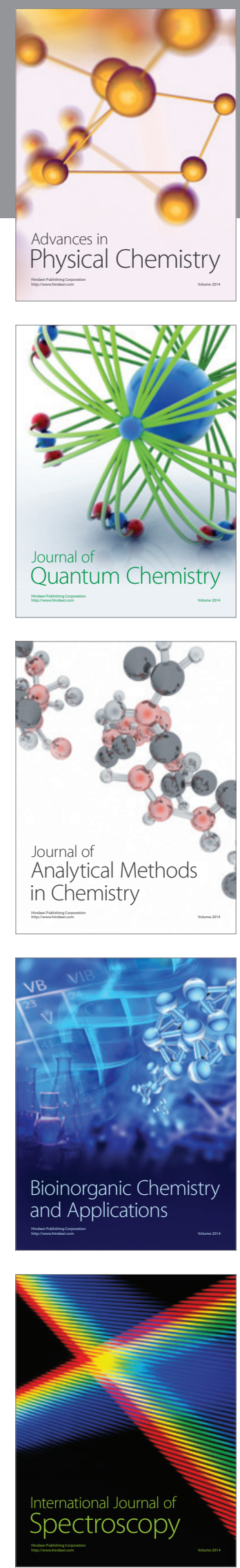